\title{
Stationary state volume fluctuations in a granular medium
}

\author{
Matthias Schröter* Daniel I. Goldman, and Harry L. Swinney \\ Center for Nonlinear Dynamics and Department of Physics \\ University of Texas at Austin, Texas 78712, USA
}

(Dated: September 29, 2018)

\begin{abstract}
A statistical description of static granular material requires ergodic sampling of the phase space spanned by the different configurations of the particles. We periodically fluidize a column of glass beads and find that the sequence of volume fractions $\phi$ of post-fluidized states is history independent and Gaussian distributed about a stationary state. The standard deviation of $\phi$ exhibits, as a function of $\phi$, a minimum corresponding to a maximum in the number of statistically independent regions. Measurements of the fluctuations enable us to determine the compactivity $X$, a temperature-like state variable introduced in the statistical theory of Edwards and Oakeshott [Physica A 157, 1080 (1989)].

PACS numbers: 45.70.-n, 05.40.-a, 64.30.+t, 47.55.Kf
\end{abstract}

Granular materials consist of a large number $N$ (typically more than $10^{6}$ ) dissipative particles that are massive enough so that their potential energy is orders of magnitude larger than their thermal energy. The large number suggests that a statistical description might be feasible. Edwards and coworkers [1] developed such a description with the volume $V$ of the system, rather than the energy, as the key extensive quantity in a static granular system. The corresponding configuration space contains all possible mechanically stable arrangements of grains.

Brownian motion is insufficient for a granular system to explore its configuration space, so energy must be supplied by external forcing such as tapping [2], shearing [3] or both [4]. The theory of Edwards requires that the forcing assures ergodicity: all mechanically stable configurations must be equally probable and accessible. A necessary condition for ergodicity is history independence: the physical properties of the system must not depend on the way a specific state was reached. History independence has previously been demonstrated only by Nowak et al. 2] for tapped glass beads at volume fractions $\phi>0.625$.

In this paper we explore the configuration space using a periodic train of flow pulses in a fluidized bed. A stationary column of glass beads in water is expanded by an upward stream of water until it reaches a homogeneously fluidized state [5], and then the flow is switched off. The fluidized bed collapses [ [] and forms a sediment of volume fraction $\phi$, which we find depends in a reproducible way on the flow rate $Q$ of the flow pulse. This forcing results in a history independent steady state where the volume exhibits Gaussian fluctuations around its average value.

A central postulate of the Edwards theory is the existence of a temperature-like state variable called compactivity $X=\partial V / \partial S$. The entropy $S$ is defined in analogy to classical statistical mechanics as $S(V, N)=\lambda \ln \Omega$, where $\Omega$ is the number of mechanically stable configurations of $N$ particles in $V$, and $\lambda$ is an unknown ana$\log$ to the Boltzmann constant. The assumption that
$X$ is a relevant control parameter in granular systems has found support in simulations of segregation in binary mixtures 7], compaction under vertical tapping [8], and shearing [9]. However, no measurements of $X$ have been reported. In this paper we determine $X$ from the measured volume fluctuations using a method suggested in [2].

Experiment. The apparatus is shown in Fig. 1(a). A square bore glass tube $(24.1 \mathrm{~mm} \times 24.1 \mathrm{~mm})$ contains about $3.6 \times 10^{6}$ beads (soda-lime glass, $d=250 \pm$

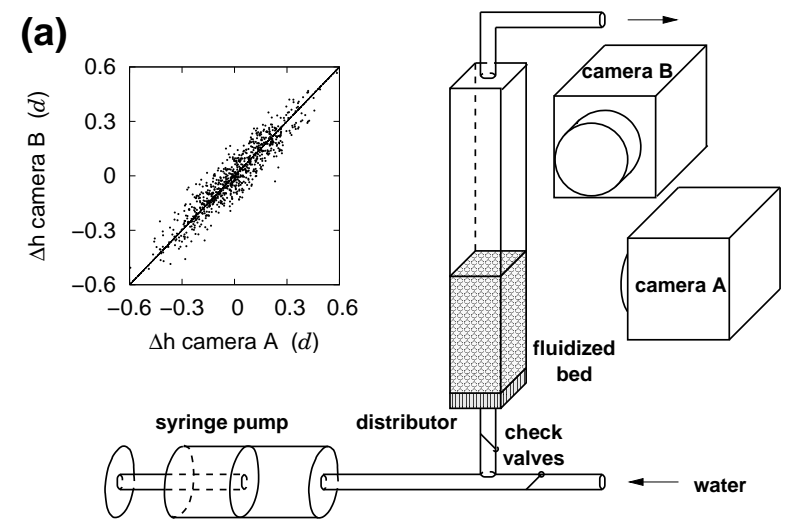

(b)

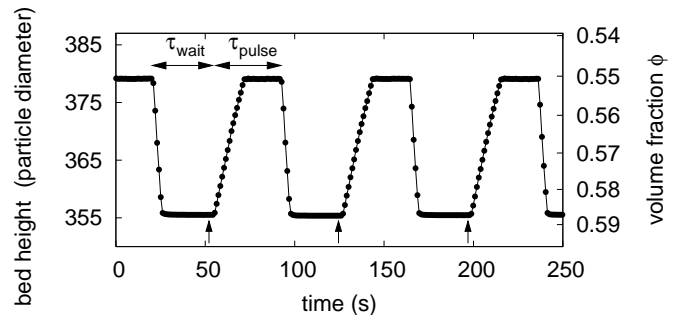

FIG. 1: (a) Experimental setup. The inset shows the correlation between the height changes $\Delta h$ during a flow pulse to the fluidized bed, as seen by the two cameras (800 flow pulses, $Q=60 \mathrm{~mL} / \mathrm{min}$ ). (b) Development of the bed height during flow pulses of $Q=40 \mathrm{~mL} / \mathrm{min}$. The arrows $(\uparrow)$ indicate measurements of $h$. 

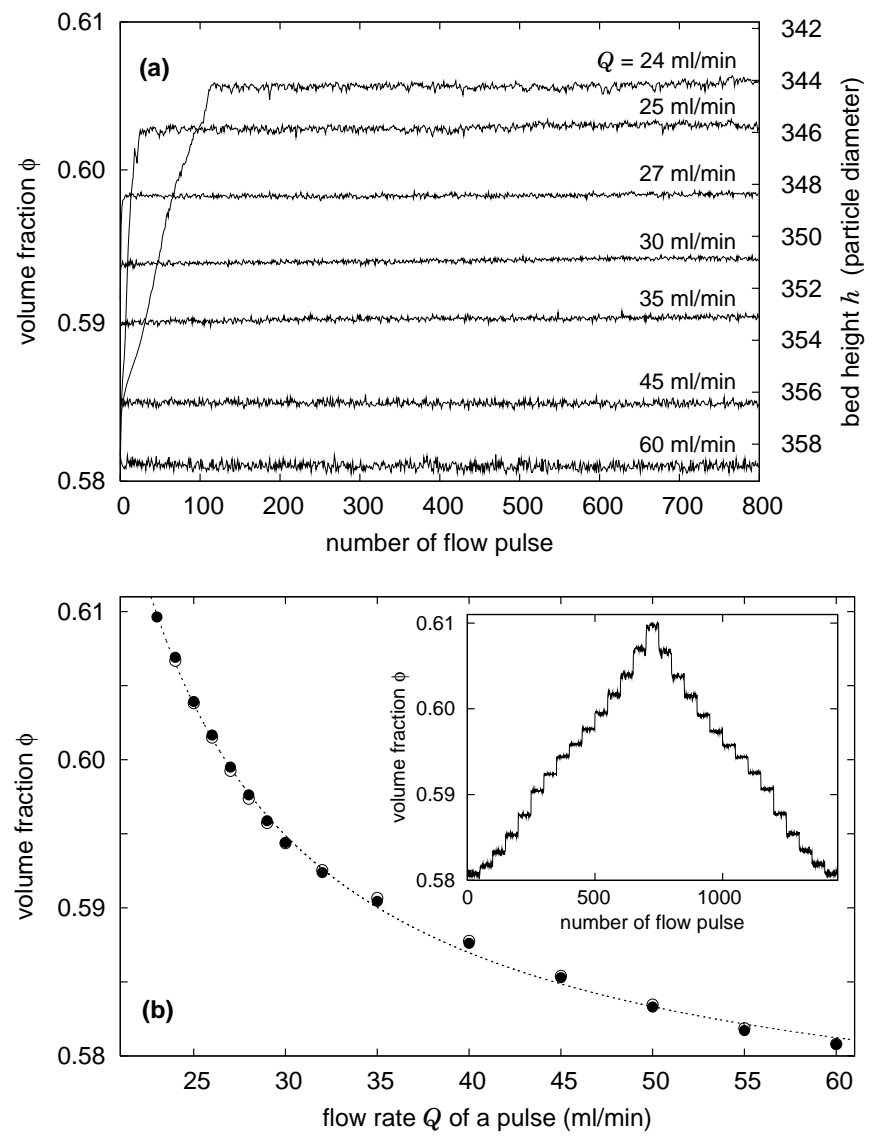

FIG. 2: History independent steady state: (a) The volume fraction $\phi$ of the sedimented bed reaches a steady state that depends only on the flow rate $Q$. (b) The inset shows the increase in volume fraction as $Q$ was decreased in 14 steps from $60 \mathrm{~mL} / \mathrm{min}$ to $23 \mathrm{~mL} / \mathrm{min}$, followed by a decrease in volume fraction as $Q$ was increased again; each step consisted of 50 flow pulses. The main graph (b) shows that the steady state volume fraction is the same for increasing (o) and decreasing $(\bullet)$; the dashed line is a fit to (1).

$13 \mu \mathrm{m}$, density $2.46 \mathrm{~g} / \mathrm{cm}^{3}$, MO-SCI Corporation). The beads are fluidized with pulses of temperature-controlled $\left(23.0 \pm 0.1^{\circ} \mathrm{C}\right)$ de-ionized water.

Flow pulses were generated by a computer-controlled syringe pump. During a flow pulse of length $\tau_{\text {pulse }}$ (Fig प(b)), the bed expanded until its height reached a stable value where the time-averaged viscous drag force on each particle corresponded to its weight; $\tau_{\text {pulse }}$ was chosen so $Q \tau_{\text {pulse }}=30 \mathrm{~mL}$. After each flow pulse, the bed was allowed to settle into a mechanically stable configuration, which was determined by measuring the timeresolved correlation of laser speckle patterns [10]. A waiting time $\tau_{\text {wait }}$ of $30 \mathrm{~s}$ was found to be ample for achieving a jammed state [11]. After $\tau_{\text {wait }}$ the volume fraction $\phi$ was determined by measuring the bed height $h$ with two CCD cameras at a $90^{\circ}$ angle. Averaging over the width of the bed yielded height values with a standard deviation
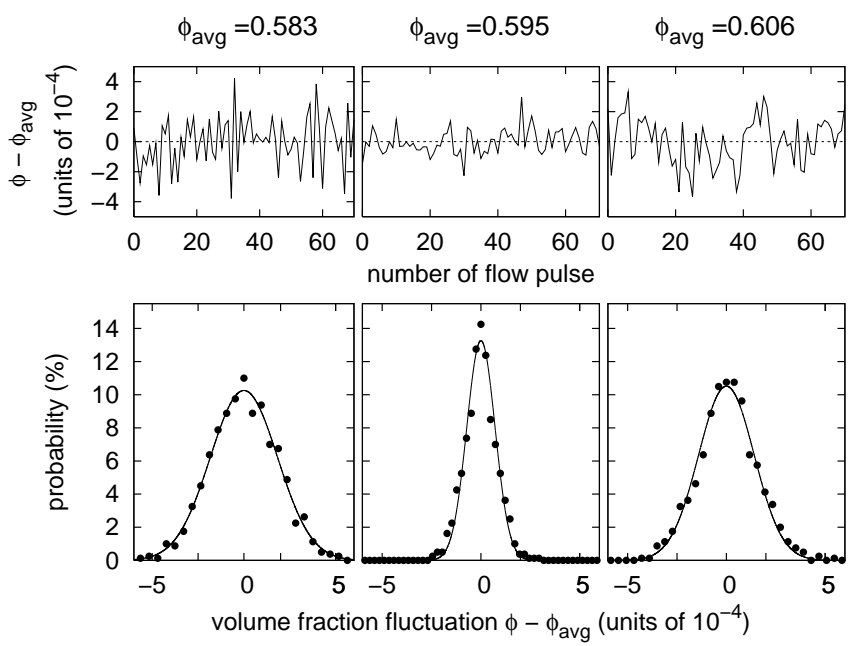

FIG. 3: Volume fraction fluctuations around the steady states shown in Fig. 2 (a) are Gaussian distributed. The upper row shows examples of the volume fraction measurements as a function of the number of flow pulses; $\phi_{\text {avg }}$ is indicated above each column. The lower row shows the corresponding probability distributions obtained from 800 flow pulses; the curves are Gaussian fits.

of only $0.008 d$ for a bed at rest (cf. inset of Fig. 囵a)).

History independent steady state. Starting with an initial $\phi=0.581$ prepared with a single flow pulse of $Q$ $=60 \mathrm{~mL} / \mathrm{min}$, the volume fraction quickly approaches a $Q$-dependent constant value $\phi_{\text {avg }}$, as shown in Fig 2 (a). Then with successive flow pulses, $\phi$ fluctuates about $\phi_{\mathrm{avg}}$. The history independence is demonstrated by ramping up and down in flow rate (Fig. 2(b)); $\phi$ depends only on $Q$ of the last flow pulse, not the earlier history of the bed. The remaining differences in $\phi_{\text {avg }}$ for increasing and decreasing $Q$ are on average only $1.6 \times 10^{-4}$. These small variations are correlated with the viscosity changes due to the temperature drift of $\pm 0.1^{\circ} \mathrm{C}$ during the course of the experiment $(35 \mathrm{~h})$.

For slow sedimentation, corresponding to $Q \rightarrow \infty, \phi$ should converge to its random loose packing value, $\phi_{\text {RLP }}$ [12]. In the absence of a theory, we fit the data to a phenomenological equation,

$$
\phi(Q)=\phi_{\mathrm{RLP}}+\frac{a}{Q-b}
$$

(Fig. 2(b)). This yields $\phi_{\mathrm{RLP}}=0.573 \pm 0.001 \quad(a=$ $0.365 \mathrm{~mL} / \mathrm{min}, b=12.9 \mathrm{~mL} / \mathrm{min}$ ), where the uncertainty arises not from the very small uncertainty in bed height measurement (corresponding to 0.0003 in $\phi$ ) but from variations in the cross section of the square tube.

Volume fluctuations. Gaussian fluctuations in $\phi$ are observed for the whole range of $\phi$ studied; examples are shown in Fig. 3 The standard deviation of these fluctuations $\sigma_{\phi}$, like $\phi_{\text {avg }}$, was found to be history independent. The variation of $\sigma_{\phi}$ with $\phi$ fits a parabola with 


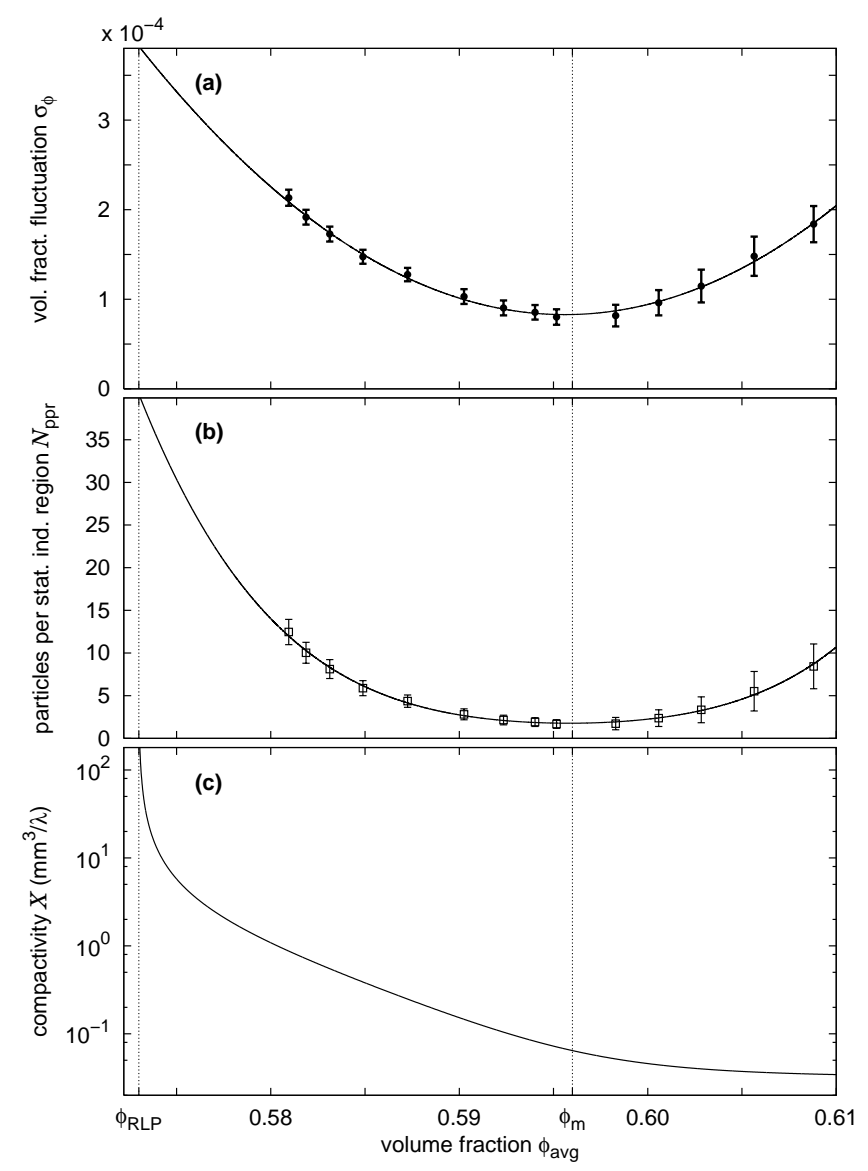

FIG. 4: (a) Volume fraction fluctuation measurements fit a parabolic curve, (b) number of particles in a statistically independent region, and (c) compactivity as a function of the average volume fraction. At $\phi_{m}$ the system has a maximum in the number of statistically independent regions (cf. (2)).

a minimum at $\phi_{\mathrm{m}}=0.596$ (Fig. 廿(a)). The minimum corresponds to a maximum in the number of statistically independent spatial regions $N_{\mathrm{i}}$ at the moment of solidification,

$$
\frac{\sigma_{\phi}}{\phi_{\mathrm{avg}}}=\frac{k}{\sqrt{N_{\mathrm{i}}}}
$$

where $k \approx 0.2$, estimated by Nowak et al. 2] from the maximal volume fraction fluctuations a single spatial region could undergo. Using (2) and the parabolic fit in Fig. 四(a), we estimate the average number of particles per statistically independent region, $N_{\mathrm{ppr}}=N / N_{\mathrm{i}}$ (where $N$ is the number of particles). Figure 4(b) shows $N_{\mathrm{ppr}}$ to be 40 for random loose packing, it reaches a minimum of about 1.8 at $\phi_{\mathrm{m}}$ and then increases again. This contrasts with the roughly constant value of $N_{\mathrm{ppr}}$ found in previous experiments [2] for $\phi$ in the range 0.625-0.634.

We suggest that the minimum of $N_{\text {ppr }}$ at $\phi_{m}$ corresponds to a crossover of competing mechanisms. With increasing $\phi$ above $\phi_{R L P}$, where force chains stabilize
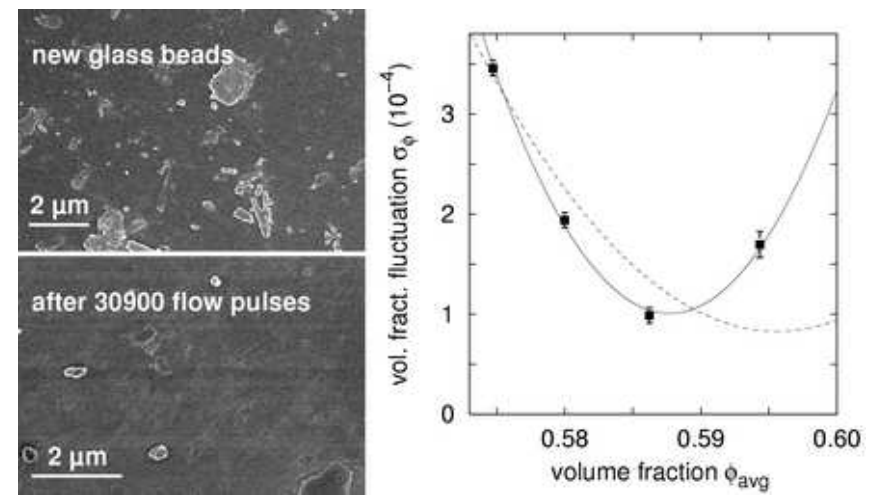

FIG. 5: Influence of particle surface roughness. On the left are scanning electron microscopy images of the surface of new and aged glass beads. The plot on the right shows the volume fraction fluctuations of new beads ( $)$ together with a parabolic fit (solid line); to minimize aging, measurements were made with only 200 flow pulses. The dashed line is the parabolic fit for aged beads from Fig. 4

the medium, the number of contacts per grain increases [13], and the probability that the displacement of one grain will mechanically destabilize other grains decreases. Consequently $N_{\text {ppr }}$ decreases. But with increasing $\phi$, the free volume per particle decreases. The latter effect is dominant for $\phi>\phi_{m}$, where geometrical constraints allow movement only as collective process of an increasing number of beads. Hence $N_{\mathrm{ppr}}$ and $\sigma_{\phi}$ increase. At the maximum random jammed packing, $\phi_{\mathrm{MRJ}}=0.64$, any motion would require a rearrangement of the whole system [14].

Knowing the dependency of $\sigma_{\phi}$ on $\phi$ enables us to determine Edward's compactivity $X$ using a granular version of the fluctuation dissipation theorem derived by Nowak et al. 2]. We obtain

$$
\frac{\lambda \rho}{m} \int_{\phi_{\mathrm{RLP}}}^{\phi}\left(\frac{\phi^{\prime}}{\sigma_{\phi^{\prime}}}\right)^{2} d \phi^{\prime}=\frac{1}{X(\phi)}
$$

where we have used $X\left(\phi_{\mathrm{RLP}}\right)=\infty$ from granular statistical mechanics, and $\rho$ is the particle density. We solve (3) numerically by substituting $\sigma_{\phi}$ from the parabolic fit (Fig. प(a)) and starting at $\phi_{\text {RLP }}$ determined from the fit with (11). This first experimental result obtained for $X$ is shown in Fig. 目(c); the values involve the unknown multiplicative factor $\lambda$. No theoretical or numerical work has predicted how $X$ depends on $\phi$. Knowing $X$ will allow further tests of granular statistical mechanics.

Aging and friction. Our experiments revealed an aging effect due to a change in the frictional properties of the beads. Friction between beads decreases with multiple collisions, which lead to a decrease in the number of

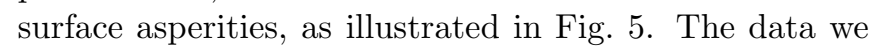
have presented show no aging during the 12,000 pulses in the experiment; the beads had been previously used 
for more than 45,000 flow pulses. Just as for aged beads, the volume fraction standard deviation for new beads fits a parabola, as Fig. 5 illustrates; the minimum is at $\phi_{\mathrm{m}}=0.587$. The shift of the minimum to a value lower than lower $\phi_{\mathrm{m}}=0.596$ for aged beads is consistent with our argument about statistically independent regions: a higher values of $\mu$ makes dilute states less fragile and lowers the onset of the necessity of collective motion. For new beads a fit to (1) results in $\phi_{\mathrm{RLP}}=0.568 \pm 0.001$, in accord with $\phi_{\mathrm{RLP}}=0.566 \pm 0.004$ interpolated from the measurements (presumably with new beads) in 12]. This shows that $\phi_{\mathrm{RLP}}$ depends on the frictional properties of the beads, as predicted in 15].

Conclusions. We have shown that the configuration space of a granular medium can, by using flow pulses, be explored in a history independent way, which is essential for the statistical theory [1] to be applicable. Fluctuations of the volume fraction are Gaussian with a parabolic minimum, which corresponds to a maximum in the number of statistically independent regions. The minimum arises as a consequence of competing mechanisms; the location of the minimum depends on the frictional properties of the beads. The compactivity can be determined from the volume fraction fluctuations; hence compactivity is a well-defined parameter representative of the material. This opens the door for new experiments that would, for example, investigate the use of compactivity as a control parameter for segregation in binary mixtures 7 , 15.

We thank Antonio Coniglio for rousing our interest in this problem; Massimo Pica Ciamarra, Karen Daniels, Sunghwan Jung, Salvatore Torquato, and Thomas Truskett for helpful discussions; and Michael Schmerling for help with the SEM. This work was supported by the Engineering Research Program of the Office of Basic Energy Sciences of the U.S. Department of Energy (Grant No. DE-FG03-93ER14312), by the Robert A Welch Foundation, and by the Office of Naval Research Quantum Optics Initiative (Grant No. N0001403-1-0639).

* Electronic address: schroeter@chaos.utexas.edu

[1] S. F. Edwards and R. B. S. Oakeshott, Physica A 157,
1080 (1989); A. Mehta and S. F. Edwards, Physica A 157, 1091 (1989); S. F. Edwards and D. V. Grinev, Phys. Rev. E 58, 4758 (1998); R. Blumenfeld and S. F. Edwards, Phys. Rev. Lett. 90, 114303 (2003); S. F. Edwards and D. V. Grinev, Granular Matter 4, 147 (2003); S. F. Edwards, J. Brujić, and H. A. Makse, in Unifying Concepts in Granular Media and Glasses, edited by A. Coniglio, A. Fierro, H. J. Herrmann, and M. Nicodemi (Elsevier, Amsterdam, 2004), pp. 9-23.

[2] E. R. Nowak, J. B. Knight, E. Ben-Naim, H. M. Jaeger, and S. R. Nagel, Phys. Rev. E 57, 1971 (1998).

[3] J.-C. Tsai, G. A. Voth, and J. P. Gollub, Phys. Rev. Lett. 91, 064301 (2003); M. Toiya, J. Stambaugh, and W. Losert, Phys. Rev. Lett. 93, 088001 (2004);

[4] K. E. Daniels and R. P. Behringer (2004), condmat/0410087.

[5] R. Jackson, The Dynamics of Fluidized Particles (Cambridge University Press, 2000); S. Sundaresan, Annu. Rev. Fluid Mech. 35, 63 (2003).

[6] D. J. Goldfarb, Ph.D. thesis, Rutgers, New Brunswick, New Jersey (2000); R. Ojha, N. Menon, and D. J. Durian, Phys. Rev. E 62, 4442 (2000); C. Gutfinger, A. Goldshtein, and H. Weinstein, to appear in Adv. Powder Techn.

[7] M. Nicodemi, A. Fierro, and A. Coniglio, Europhys. Lett. 60, 684 (2002); A. Prados and J. J. Brey, Europhys. Lett. 64, 29 (2003); A. Coniglio, A. Fierro, and M. Nicodemi, Physica D 193, 292 (2004).

[8] A. Barrat, J. Kurchan, V. Loreto, and M. Sellitto, Phys. Rev. Lett. 85, 5034 (2000); A. Fierro, M. Nicodemi, and A. Coniglio, Europhys. Lett. 59, 642 (2002); J. J. Brey and A. Prados, Physical Review E 68051302 (2003); D. S. Dean and A. Lefèvre, Phys. Rev. Lett. 90, 198301 (2003); G. Tarjus and P. Viot, Phys. Rev. E 69, 011307 (2004).

[9] H. A. Makse and J. Kurchan, Nature 415, 614 (2002).

[10] L. Cipelletti, H. Bissig, V. Trappe, P. Ballesta, and S. Mazoyer, J. Phys.: Condens. Matter 15, S257 (2003).

[11] A. J. Liu and S. R. Nagel, Nature 396, 21 (1998).

[12] G. Y. Onoda and E. G. Liniger, Phys. Rev. Lett. 64, 2727 (1990).

[13] L. E. Silbert, D. Ertaş, G. S. Grest, T. C. Halsey, and D. Levine, Phys. Rev. E 65, 031304 (2002).

[14] S. Torquato, T. M. Truskett, and P. G. Debenedetti, Phys. Rev. Lett. 84, 2064 (2000); A. R. Kansal, S. Torquato, and F. H. Stillinger, Phys. Rev. E 66, 041109 (2002); A. Donev, S. Torquato, and F. H. Stillinger, Phys. Rev. E 71, 011105 (2005).

[15] Y. Srebro and D. Levine, Phys. Rev. E 68, 061301 (2003). 